\title{
Die Schlei, ein Modell für die Verbreitung der Strandkrabbe Carcinus maenas
}

\author{
M. Dries \& D. Adelung* \\ Institut für Meereskunde an der Universität Kiel; \\ Düsternbrooker Weg 20, D-2300 Kiel, Bundesrepublik Deutschland
}

\begin{abstract}
The Schlei as a model for the distribution of the green shore crab Carcinus maenas. A Carcinus maenas population inhabiting the Schlei, a glacial fjord of the Baltic Sea, was studied during a three-year period of at least monthly sampling. Due to slightly higher water temperatures in the Schlei (c. $1^{\circ} \mathrm{C}$ higher than in the neighbouring waters of the western Baltic Sea) annual larval development starts there one month earlier. When in some years salinities are unfavourable $(<13 \%)$, larval development may be almost completely prevented. Juveniles and adults tolerate changing salinities, even though females prefer staying in deep waters and juveniles in shallow waters of high salinities. During winter all crabs move to deeper waters and stay huddled together in crevices and holes until March or April. Females usually moult after being fertilized, which takes place after the breeding season in August. Males moult between May and June; juveniles continue to moult during the warm season. Moulting for growth lasts until puberty is reached in the second year. From then on intermoult periods are more extended, but males moult more frequently than females, attaining ultimately a larger size. Under favourable environmental conditions, the maximum lifespan of C. maenas in the Schlei amounts to five years. During this period, five larval moults and about fifteen moults for growth occur.
\end{abstract}

\section{EINLEITUNG}

Die kosmopolitische Verbreitung der Strandkrabbe Carcinus maenas L. weist auf eine große Toleranz gegenüber verschiedenen Umweltfaktoren hin. In vielen Teilen ihres Verbreitungsgebietes, so auch in der westlichen Ostsee, stellt sie die beherrschende Art der Großkrebse im küstennahen Makrobenthos dar.

Die Frage, welche Faktorenkombinationen diese Verbreitung beschränken, ist bisher nur unzureichend beantwortet. Neben der Temperatur dürfte der Salzgehalt, zumindest in den salzärmeren Gebieten, die Verbreitung begrenzen. So ist in der Ostsee C. maenas nur bis zu einem Salzgehaltsminimum von $7 \%$ anzutreffen (Schellenberg, 1928). Aufgrund von Literaturangaben (Braun, 1888; Thorson, 1946; Kändler, 1952; Muus, 1967; Schulz, 1969; Rasmussen, 1973) ergibt sich für die Ostsee das in Abb. 1 dargestellte Vorkommen.

Salzgehalte bis zu $4 \%$ werden vorübergehend toleriert (Broekhuysen, 1937), aber um länger in einem salzgehaltsarmen Gebiet leben zu können, sind nach Ropes (1968) mindestens $11 \% \mathrm{~S}$ erforderlich. Für die dauerhafte Besiedlung sind aber die Bedingungen entscheidend, die die Fortpflanzung und die Entwicklung der empfindlichen Larven gewährleisten.

*Korrespondenzadresse 
In einem so großräumigen Biotop wie der Ostsee ist es aber kaum möglich, den Einfluß der einzelnen Faktoren auf die Entwicklung einer einzigen Population zu erfassen. Hier bietet sich die Schlei, eine eiszeitliche Förde, als natürliches, weitgehend abgeschlossenes Modell der Ostsee an, da ihre Salinitätsverhältnisse (horizontaler Salzgehaltsgradient und starke Salzgehaltsschwankungen) die Bedingungen der Ostsee widerspiegeln. Wegen der nur $25 \mathrm{~m}$ breiten Offnung ist ein Austausch der Faunenelemente mit der Ostsee nur bedingt möglich. Die Schlei erstreckt sich an der schleswigholsteinischen Ostküste $42 \mathrm{~km}$ weit in das Landinnere. Über eine Schwelle von 3,5 m Tiefe gelangt Wasser aus der Ostsee in die Schlei und führt im vorderen Teil in Abhängigkeit von den gerade herrschenden Bedingungen in der Ostsee zu Salzgehalten von 12 bis $22 \%$. Landeinwärts sinkt der Salzgehalt bis zu unter $5 \%$.

Trotz der wechselhaften und z.T. extremen Bedingungen scheint die Schlei für die Strandkrabbe ein günstiger Lebensraum zu sein. Sie kommt hier häufig in so großen Mengen vor, daß sie für die örtliche Fischerei zeitweise eine ernsthafte Plage darstellt. Jedoch geht aus den Fangerträgen der Fischer hervor, daß die Populationsdichte von $C$. maenas in der Schlei stark schwankt. Dies weist darauf hin, daß die Schlei ein Grenzgebiet für die Verbreitung darstellt und daher die Verbreitungsfaktoren von C. maenas besonders gut zu untersuchen sind.

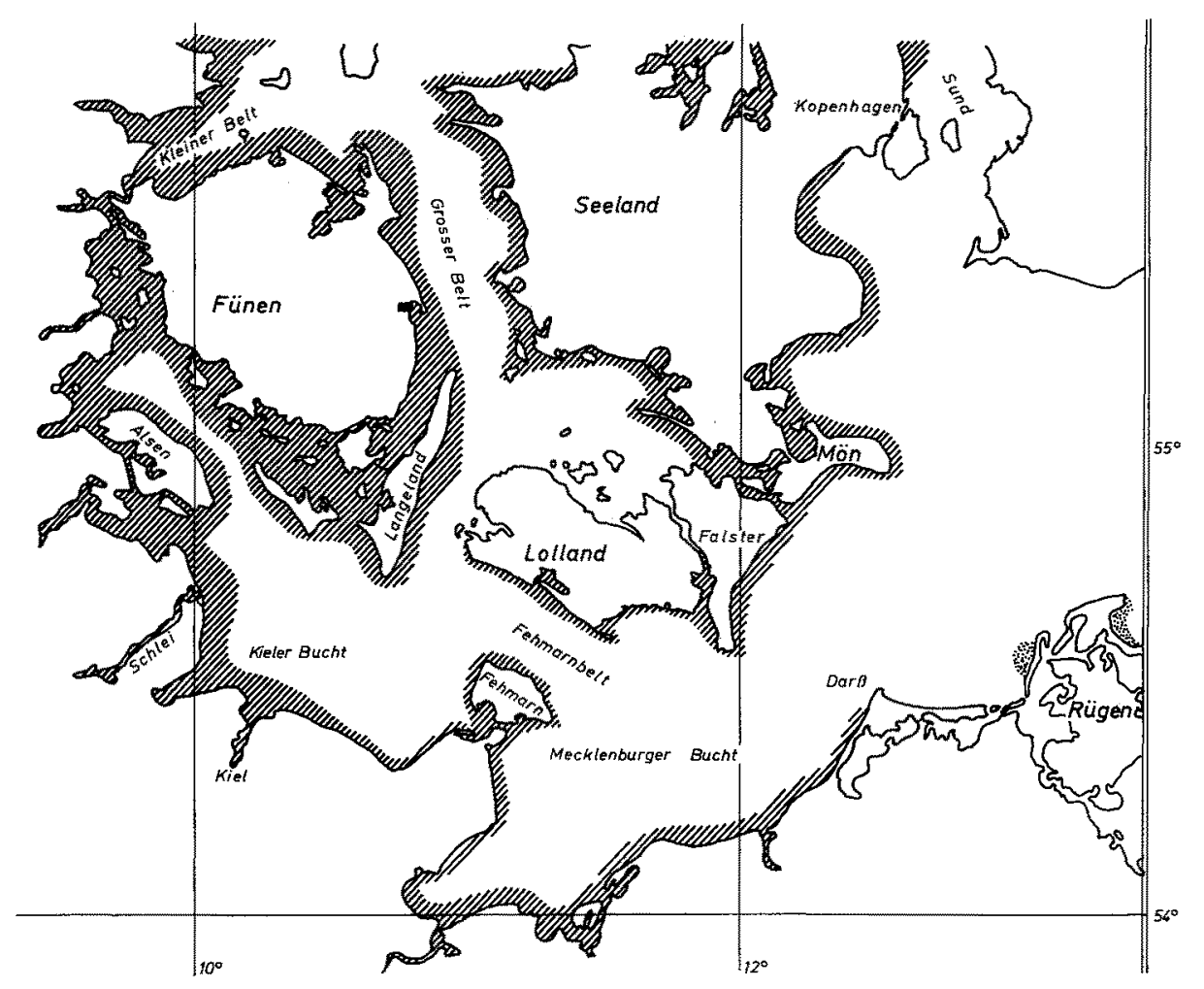

Abb. 1. Verbreitung (gestrichelt) von Carcinus maenas in der Kieler Bucht und Westlichen Ostsee (zusammengestellt nach Literaturangaben) 


\section{MATERIAL UND METHODEN}

Von Oktober 1973 bis September 1976 fanden in der Regel einmal monatlich, in den Sommermonaten mehrmals Untersuchungen in der äußeren Schlei, im wesentlichen im Gebiet von Schleimünde bis Kappeln statt. Auf Abb. 2 sind die Stationen (ca. 1 Meile Abstand) markiert, bei denen jeweils Salzgehalt und Temperatur mittels einer TS-Sonde an der Oberfläche und über dem Boden gemessen wurden. Auf den Strecken zwischen den Stationen wurde der Boden mit einer kleinen Baumkurre $(2,8 \mathrm{~m}$ Baumlänge, $7 \mathrm{~mm}$ Maschenweite) abgefischt. Bodenbeschaffenheit, Vegetation und besonderer Beifang wurden notiert, alle Strandkrabben aussortiert und ihre Carapaxbreite bis auf $0,5 \mathrm{~mm}$ genau vermessen. Größere Krabben (über $15 \mathrm{~mm}$ Carapaxbreite) wurden nach Geschlecht getrennt ausgezählt und alle sichtbaren biologischen Gegebenheiten wie Häutung, Kopulation und Trächtigkeit protokolliert.

In den Wintermonaten 1974/75 und 1975/76 erfolgte eine genauere Erforschung des äußeren Schleigebietes bei Schleimünde mit Hilfe der Scuba-Tauchtechnik an der in Abb. 2 gekennzeichneten Stelle. Dabei wurden folgende Arbeiten durchgeführt: Fang der Strandkrabben mit der Hand aus 5 bis $7 \mathrm{~m}$. Tiefe, Vermessung des Fanggebietes, Entnahme von Bodenproben (Erdklumpen mit Infauna), die sofort in verschließbare Plastikbehälter kamen, Fotografieren der Unterwassersituation.

Eine genauere Analyse der Bodenproben erfolgte im Laboratorium. Es wurde die Zusammensetzung der Endofauna bestimmt, alle darin enthaltenen Strandkrabben gemessen und ihre Bestandsdichte auf $1 \mathrm{~m}^{2}$ Bodenfläche mit $10 \mathrm{~cm}$ Schichttiefe berechnet. Eine genauere Bestimmung der Bodenbeschaffenheit erfolgte im Geologischen Institut der Universität Kiel.

Um auch die Larven zu erfassen, wurden in den Sommern 1975 und 1976 mit Planktonnetzen $(40 \mathrm{~cm} \varnothing, 335 \mu \mathrm{m} ; 80 \mathrm{~cm} \varnothing, 200 \mu \mathrm{m})$ in und vor der Schlei an festgeleg-

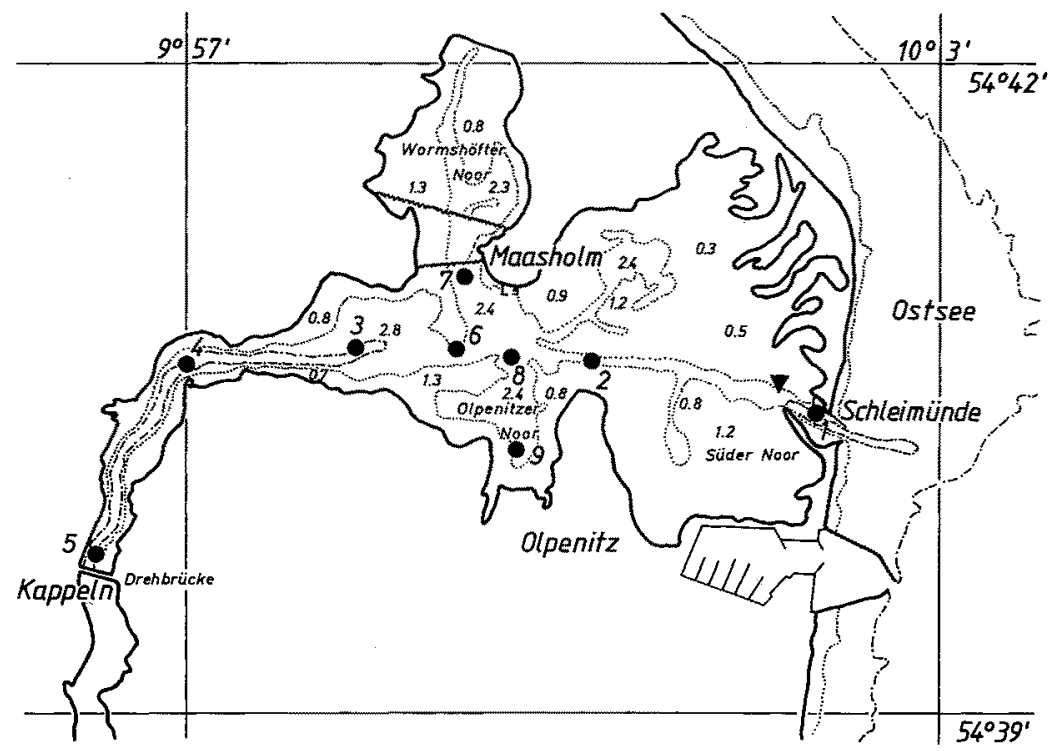

Abb. 2. Untersuchungsgebiet der äußeren Schlei von Schleimünde bis Kappeln mit Tiefen- und Stationsangaben: ...... $2 \mathrm{~m}$ Tiefenlinie, $5 \mathrm{~m}$ Tiefenlinie, $\bullet$ Meßstationen, $\nabla$ Tauchstation 
ten Stationen horizontal und vertikal Planktonproben gezogen. Die Fänge erfolgten jeweils zwischen 11 und 14 Uhr. Das Plankton wurde sofort in $4 \%$ igem Formol fixiert und später die darin enthaltenen Carcinus-Larven ausgezählt.

Zur statistischen Absicherung der Ergebnisse wurden der t-Test und der U-Test von Wilcoxon, Mann \& Whithney angewendet.

\section{ERGEBNISSE}

Wie eine Literatur- und Datenstudie über Salzgehalt, Temperatur sowie die Wetterverhältnisse ergab, werden die hydrographischen Verhältnisse in der Schlei durch diejenigen der Ostsee bestimmt.

\section{Salzgehalt}

In den Jahren 1974, 1975 und 1976 waren in dem Gebiet zwischen Schleimünde und Kappeln übereinstimmend die Salzgehalte im Sommer geringer als im Winter, jedoch unterschieden sich die Werte in den einzelnen Sommern beträchtlich. 1974 betrug der Salzgehalt im Juni und Juli durchschnittlich 12 bis 13\%, 1976 im gleichen Zeitraum um $15 \%$.

Im Winter stieg der Salzgehalt in allen drei Untersuchungsjahren an: Im Jahre 1973/ 74 , soweit Daten vorliegen, bis auf maximal $17 \%$; $1974 / 75$ bis auf ca. $19 \%$ und 1975/76 sogar bis auf ca. $22 \%$ S. In der Schlei unterschieden sich der Salzgehalt des Oberflächenwassers und des Bodenwassers maximal um weniger als $1 \%$.

\section{Temperatur}

Die drei Untersuchungsjahre zeigten folgende Temperaturverhältnisse: In jedem Jahr erwärmte sich das Wasser in dem Untersuchungsgebiet im Monat Mai auf über $10^{\circ} \mathrm{C}$. Die maximalen Temperaturen wurden im Juni/Juli erreicht, 1974 waren die Sommertemperaturen niedriger als in den Jahren 1975 und 1976, in denen die Wassertemperatur über $20^{\circ} \mathrm{C}$ anstieg. Insgesamt waren die Oberflächentemperaturen in der Schlei während des Sommers im Mittel um $1,2^{\circ} \mathrm{C}$ höher als in dem angrenzenden Ostseegebiet.

In den Wintermonaten wurden in allen drei Untersuchungsjahren niemals Temperaturen unter $0^{\circ} \mathrm{C}$ gemessen. Diese Winter sind daher als relativ mild einzustufen.

\section{Bodenvegetation}

Die Schlei ist ein hochgradig eutrophiertes Gewässer, dem ständig neue Nährstoffe und fäulnisfähige organische Substanzen durch Abwassereinleitung und Bodendüngung zugeführt werden. Schon bei Schleimünde weist die Schlei eine verhältnismäßig starke Eutrophierung auf (vgl. Rheinheimer, 1970). In der äußeren Schlei, vor allem außerhalb der Fahrwasserrinne, ist eine reiche Bodenvegetation vorhanden, die in Richtung Kappeln abnimmt. In diesen Bewuchszonen waren viele kleine und junge Tiere wie Fischbrut, Gammariden, SeenadeIn und juvenile Strandkrabben zu finden. 


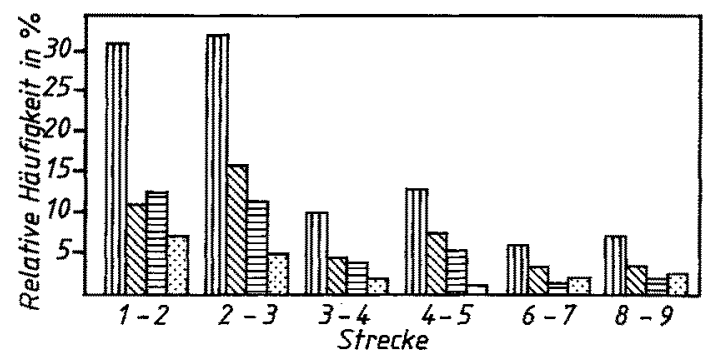

Abb. 3. Die prozentuale Verteilung der Fangergebnisse auf den einzelnen Fangstrecken in der Schlei, bezogen auf deñ gesamten Fangertrag von Oktober 1973 bis September 1976. Die einzelnen Balken stellen die prozentualen Anteile aller Tiere (Längsschraffur), der $\delta \delta$ (Schrägschraffur), der

우 (Querschraffur) und der Juvenilen unter $20 \mathrm{~mm}$ Carapaxbreite (Punktraster) dar

\section{Die Verbreitung von Carcinus maenas in der Schlei}

\section{Larvalperiode}

Um festzustellen, ob in der Schlei eine eigene Population vorhanden ist oder sich der Bestand aus der Ostsee rekrutiert, wurden 1976 Planktonfänge in der Schlei und in dem Ostseegebiet vor der Schlei unternommen. Diese ergaben in der Schlei ein dichteres und früheres Larvenaufkommen als vor der Schlei. Im äußeren Schleigebiet bei Schleimünde und in Richtung Olpenitzer Noor wurden die meisten Larven gefangen. Larven des ersten Zoeastadiums wurden erstmals am 24.6. 1976 gefunden. Nach einem schnell erreichten Maximum Ende Juni nahm in der Schlei die Zahl der Larven mit fortschreitender Jahreszeit bis zum September ab. Lediglich Ende August konnte ein kurzfristiger Zwischenanstieg in der Larvenzahl registriert werden. Dies weist auf eine zweite Brutperiode hin.

In der Ostsee lag das Fangmaximum etwa einen Monat später als in der Schlei. Vermutlich bedingt die langsamere Erwärmung der Ostsee diese gegenüber der Schlei verzögerte Brutzeit.

Insgesamt scheinen die Larven der Schlei bessere Futterbedingungen vorzufinden als in der Ostsee, da bei $50 \%$ der gefangenen Larven aus der Schlei der Darm gefüllt war, aber nur $5 \%$ der Larven aus der Ostsee Darminhalt aufwiesen.

Die Ergebnisse belegen, daß C. maenas in der Schlei seine vollständige Larvalentwicklung durchlaufen kann und die Population nicht auf Erneuerung aus der Ostsee angewiesen ist.

\section{Zusammensetzung, Verteilung und Veränderung der Population juveniler und adulter Strandkrabben}

Aufgrund der hydrographischen Verhältnisse ist eine ungleichmäßige Verteilung der Strandkrabben in der Schlei zu erwarten. Um dies zu überprüfen, wurden alle Fänge (insgesamt 6346 Tiere) in dem gesamten Beobachtungszeitraum von Oktober 1973 bis September 1976 ausgewertet. Wie aus Abbildung 3 hervorgeht, kommen in dem vorderen (Strecke 1 bis 2) und mittleren Abschnitt (Strecke 2 bis 3) des Untersuchungsgebietes die Strandkrabben dreimal häufiger vor als in den salzärmeren hinteren Gebieten (Strecke 3 bis 4 und 4 bis 5). Noch geringer ist die Verbreitung in den Flachwassergebieten des mittleren Abschnittes (Strecke 6 bis 7 und Strecke 8 bis 9). 
Allerdings verteilen sich die Männchen, Weibchen und Juvenilen auf den verschiedenen Fangstrecken unterschiedlich; so überwiegen die Weibchen im Streckenabschnitt 1 bis 2, während in den anderen Abschnitten, insbesondere im hinteren Abschnitt und in den Flachwassergebieten die Männchen weit über die mittlere Geschlechtsverteilung hinaus vorherrschen.

Das Vorkommen der Juvenilen entspricht in den vorderen Abschnitten dem Gesamtmittel von $18 \%$, im hinteren Abschnitt ist es mit nur 4,3\% sehr gering. In den flachen Nebenstrecken sind dagegen die juvenilen Tiere doppelt so stark wie im Gesamtmittel vertreten. Daraus läßt sich schließen, daß für die Weibchen die Lebensbedingungen vor allem in den salzreicheren, tiefen Gebieten und für die Juvenilen in den salzreicheren Flachwassergebieten am geeignetesten sind. Die Männchen stellen nach der Verteilung offenbar die geringsten Ansprüche an ihren Lebensraum.

Berücksichtigt man die jährlichen Veränderungen, so ergeben sich trotz gleicher Fangtechnik erhebliche Unterschiede in der Besiedlungsdichte: Im Jahre 1974 wurden bei 9 Ausfahrten insgesamt nur 374, 1975 bei 13 Ausfahrten 3116 und 1976 bei 13 Ausfahrten 2793 Tiere gefangen. Das bedeutet ein starkes Ansteigen der Populationsdichte von 1974-1975.

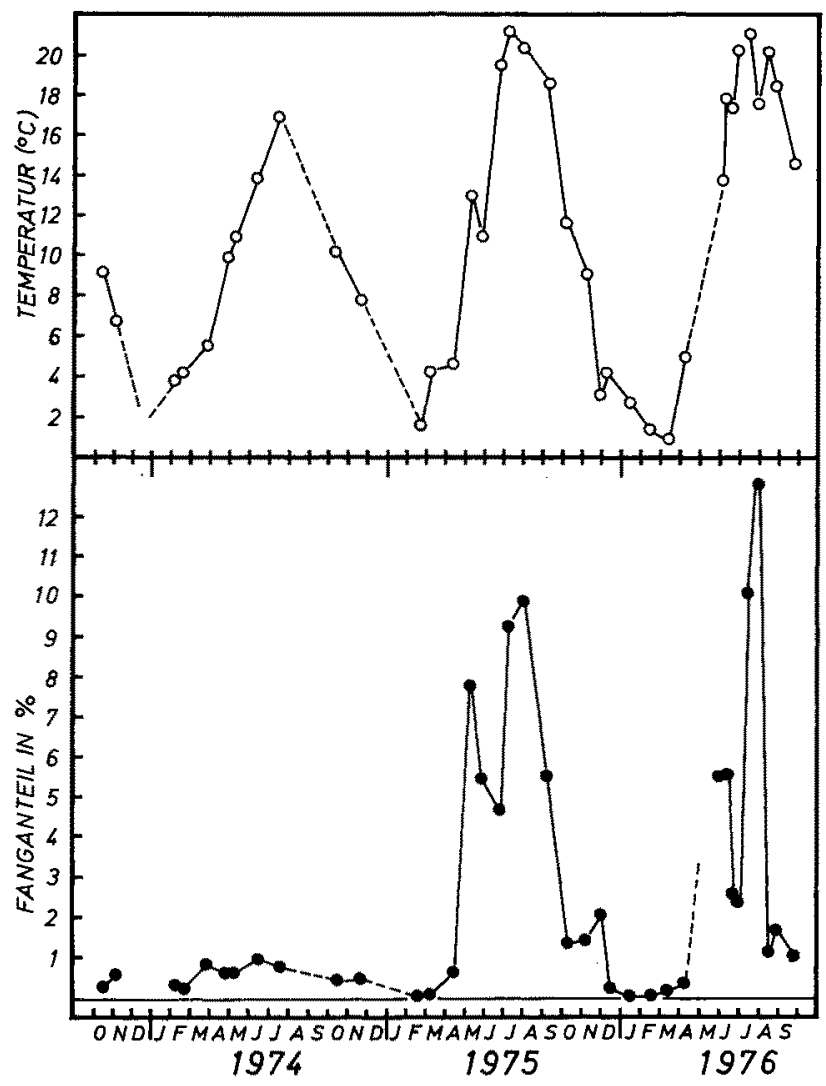

Abb. 4. Fanganteile der einzelnen Monate in Prozent, bezogen auf den Gesamtfang und die Wassertemperatur während der gesamten Fangzeit 


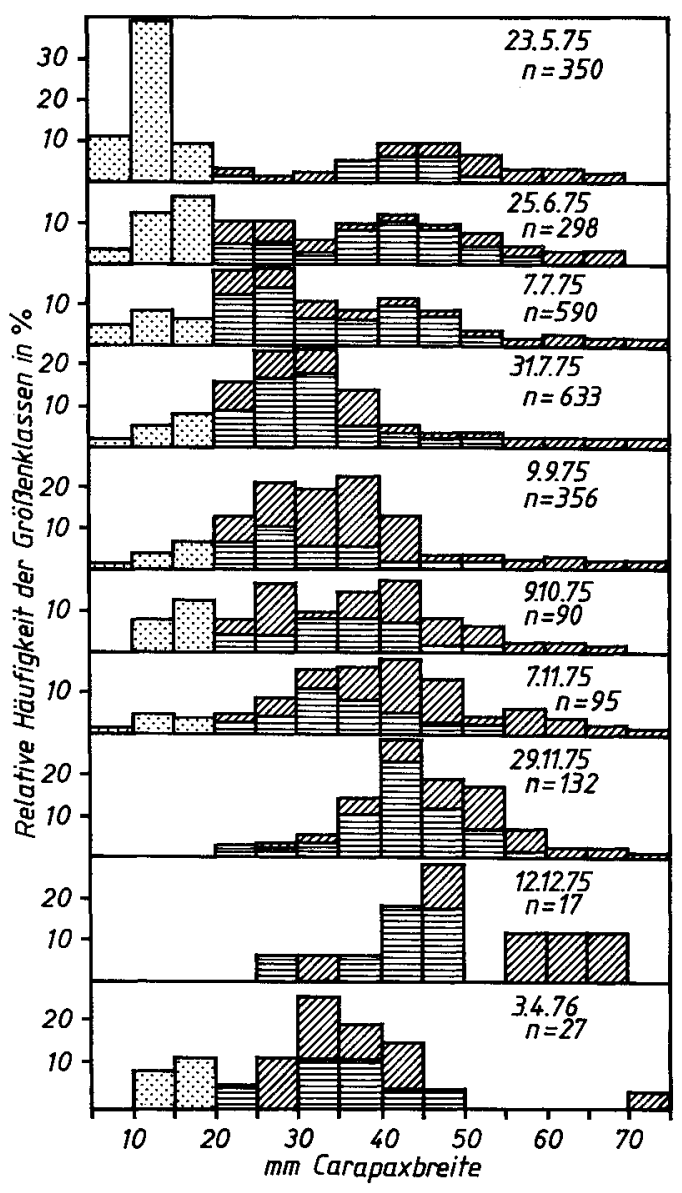

Abb. 5. Relative Häufigkeit der Größenklassen pro Fangtag in Prozent des Gesamtfanges für den Zeitraum Mai 1975-April 1976: Juvenile (Punktraster), +9 (Querschraffur), ơ (Schrägschraffur)

Jahreszeitlich verändern sich die Fangerträge in enger Beziehung zu der Wassertemperatur (vgl. Abb. 4): Bei niedrigen Temperaturen verschwinden die Strandkrabben aus dem Fanggebiet, um es bei steigender Temperatur im Frühjahr erneut zu besiedeln. In den Wintermonaten Januar und Februar konnten praktisch keine Tiere gefangen werden. Auch im April waren die Fangquoten sehr niedrig, stiegen dann aber im Mai steil an. Herausragend war das Jahr 1975 mit einem massenhaften Auftreten juveniler Tiere in diesem Monat. Während in den darauffolgenden Monaten die Zahl der Juvenilen sich wieder verminderte und in den Dezemberfängen keine Krebse unter $20 \mathrm{~mm}$ Carapaxbreite mehr vorhanden waren, nahm die Zahl der adulten Tiere ab Mai ständig zu und erreichte in den Monaten Juli und August ihr Maximum. Zum Winter hin nahmen dann auch die Fangzahlen der Adulten rasch ab.

Die Frage nach dem Verbleib der Krebse in den Wintermonaten Dezember bis März, in denen keine Krebse mehr gefangen wurden, ließ sich durch die Unterwasserarbeiten klären. Näher untersucht wurde ein Gebiet am Rande der Fahrrinne ( $\boldsymbol{\nabla}$ auf Abb. 
2). Der Hang der Fahrrinne, der hier bis auf 6-7 m Tiefe abfällt und aus Gyttja besteht, wird von einem Spaltensystem unterbrochen, das parallel zur Fahrrinne über eine Strecke von mindestens $30 \mathrm{~m}$ zu verfolgen war. Die Spalten hatten an den Öffnungen eine Höhe von $10 \mathrm{~cm}$ bis ca. $1 \mathrm{~m}$, verjüngten sich nach hinten und waren $\mathrm{z} . T$. über $1 \mathrm{~m}$ tief.

Diese Spalten waren in den Monaten Januar und Februar 1975 dicht mit Strandkrabben gefüllt. Die bei Temperaturen unter $5^{\circ} \mathrm{C}$ äußerst trägen Tiere konnten leicht von Hand eingesammelt werden. Nach der Fangzahl und auf Grund von Unterwasserfotografien des Systems errechnet sich die Besatzdichte auf $1 \mathrm{~m}$ Spaltenlänge bei $1 \mathrm{~m}$ Spaltentiefe mit 1000 bis 2000 Tieren, so daß auf $10 \mathrm{~m}$ Spaltenlänge mehr als 10000 Strandkrabben überwintern können. Da die Tiefe der größeren Höhlungen nicht zu bestimmen war, ist es durchaus möglich, daß noch mehr Tiere darin verborgen waren. Im Sommer hielten sich nur einzelne Krebse im Spaltensystem auf.

Die Beobachtungen zeigten auch eine bestimmte Anordnung der Krebse in den Spalten. Am Höhleneingang befanden sich vorwiegend große Männchen, während sich nach hinten zu kleinere Tiere aufhielten und der Anteil der Weibchen zunahm.

Die juvenilen Tiere fanden besonders gute Unterschlupfmöglichkeiten in dem von Bodenevertebraten durchlöcherten Sediment. Im März 1975 wurden in einer Bodenprobe von ca. $4000 \mathrm{~cm}^{3} 40$ Jungtiere unter $15 \mathrm{~mm}$ Carapaxbreite gefunden. Auf eine Bodenschicht von $1 \mathrm{~m}^{2}$ und $10 \mathrm{~cm}$ Dicke berechnet, kommen danach 800 bis 1000 juvenile Strandkrabben.

Die Größenanalyse aller Tiere ergibt in den Jahren 1974, 1975 und 1976 eine unterschiedliche Größenhäufigkeitsverteilung (vgl. Ab. 5): Wie schon erwähnt, stellen die Juvenilen der Größenklasse 10-15 mm Carapaxbreite von März bis Mai 1975 prozentual den größten Anteil an dem Gesamtfang. Bei ihnen handelt es sich um die Nachkommen des Jahres 1974. An ihnen ließ sich die Populationsentwicklung im Freiland gut verfolgen.

In den Monaten Mai und Juli lassen sich zwei unterschiedlich große Häufigkeitsmaxima feststellen. Das erste Maximum wird von den juvenilen Tieren gebildet, die im Juni nach zwei bis drei Häutungen bereits auf eine Größe bis zu $30 \mathrm{~mm}$ heranwachsen und geschlechtsreif geworden sind. Das zweite kleinere Maximum liegt bei Tieren mit einer Größe von 40-50 mm und wird von dem Restbestand der adulten Tiere aus dem Jahre 1974 und früher gebildet. Dieser kann noch bis zum 7 . Juli 1975 verfolgt werden und verschwindet dann aus den Fängen.

In den Monaten Juli bis September 1975 erreicht die 74iger Generation eine Carapaxbreite von 30-40 mm, wobei es sich bei den kleineren Tieren um Weibchen, bei den größeren um Männchen handelt. Dieser Größenzuwachs bedeutet, daß sich die Tiere ein weiteres Mal gehäutet haben.

Da sich aber das Größenmaximum bis in den November hinein noch weiter bis zu einer Größe von $45 \mathrm{~mm}$ verschiebt, ist mit noch einer Häutung zu rechnen, so daß sich die 74iger Generation 1975 insgesamt 4- bis 5 mal gehäutet haben dürfte.

Auch im Jahre 1976 dominiert die 74iger Generation und kann das ganze Jahr hindurch gut verfolgt werden. Im März bis Juni 1976 tritt noch ein kleines Nebenmaximum auf. Dieses wird aus juvenilen Tieren des offensichtlich kleinen Jahrgangs 1975 gebildet.

Das Größenmaximum der 74iger Generation verschiebt sich im Jahre 1976 nur noch 
Tabelle 1. Der prozentuale Anteil frischgehäuteter Männchen bzw. frischgehäuteter Weibchen (ohne Berücksichtigung der trächtigen Weibchen) am jeweiligen Tagesfang im Jahr 1976. Eingeklammerte Zahl: vorwiegend juvenile Tiere

\begin{tabular}{|rcc|}
\hline Datum & $\delta \delta^{\ddagger}(\%)$ & $\%(\%)$ \\
\hline 3.6. & 49,2 & $(20,8)$ \\
24.6. & 5,7 & 8,0 \\
29.6. & 3,5 & 17,9 \\
15.7. & 1,3 & 12,4 \\
30.7. & 0,5 & 30,9 \\
13.8. & - & 31,8 \\
27.8. & - & 17,2 \\
28.9. & - & 1,1 \\
\hline
\end{tabular}

wenig. Vom Juni bis September 1976 erreicht es die 55-mm-Größe. Diese Größenverschiebung entspricht einer einzigen Häutung. Allerdings wurden auch einige Männchen mit einer Größe bis zu $70 \mathrm{~mm}$ gefangen. Bei diesen Tieren handelt es sich wahrscheinlich um Reste der 73iger Generation.

Die unterschiedliche Größe beider Geschlechter ist darauf zurückzuführen, daß die Weibchen schon gleich bei Beginn der Geschlechtsreife begattet werden und sich während der Phase ihrer Trächtigkeit nicht häuten und damit auch nicht wachsen.

In der Zeit von Ende Mai bis Ende Juni 1976 waren $80 \%$ der gefangenen Weibchen trächtig. Im Juli sank der Prozentsatz unter $10 \% \mathrm{ab}$. Die Hauptlaichzeit für C. maenas liegt also in dem Untersuchungsgebiet in den Monaten Mai bis Juli, wobei offenbar manche Weibchen zweimal hintereinander ablaichen, wie Markierungsversuche und Laborbefunde zeigten. In Übereinstimmung mit den Laboratoriumsbefunden konnte im Freiland niemals ein Ablaichen bei Wassertemperaturen unter $10^{\circ} \mathrm{C}$ beobachtet werden.

In Tabelle 1 ist der Prozentsatz der Männchen und Weibchen aufgeführt, die im Jahre 1976 von Juni bis September in den jeweiligen Fängen in frischgehäutetem Zustand angetroffen wurden. Aus der Tabelle geht deutlich hervor, daß die Häutungsaktivität der Männchen vor allem im Zeitraum Mai bis Juni liegt und während des Juli stark zurückgeht, während die adulten weiblichen Tiere erst Ende Juli und im August ihre Haupthäutungsphase haben, also nach der Hauptlaichzeit.

\section{DISKUSSION}

Wie die unterschiedlichen Fangergebnisse in den einzelnen Jahren zeigen, stellt die Schlei für Carcinus maenas ein Grenzgebiet der Verbreitung dar. Trotz zeitweise ungenügender Fortpflanzungsbedingungen bietet sie den Tieren jedoch insgesamt günstige Lebensmöglichkeiten. Sie zeigt aber auch deutlich die Grenzen für die Verbreitung dieser Tiere in Brackwassergebieten auf.

Dank der weitgehend abgeschlossenen Lage kann man in der Schlei die Entwicklung einer Population von der Larvenphase bis in das späte Adultstadium hinein verfolgen. Voraussetzung hierfür ist allerdings, daß wie 1974 ein Jahr durch eine große Nachkommenschaft ausgezeichnet ist, während die vorangegangenen und nachfolgen- 
den Jahre nur kleine Generationen aufweisen. Bereits im Oktober und November 1974 waren die Jungtiere, die bis September ihre Larvenphase beendet hatten, mit einer durchschnittlichen Größe von $7,5 \mathrm{~mm}$ nach 6 bis 7 postlarvalen Häutungen in den Fängen stark vertreten. Im Mai 1975 erschienen diese Jungtiere in etwa gleicher Menge und in der gleichen Größenklasse. Daraus folgt, daß sie sich im Winter nicht gehäutet hatten und der Bestand durch den Winter nicht verringert worden war. Anhand der Größenhäufigkeitsverteilung ließ sich das Wachstum dieser Tiere 1975 gut verfolgen. Dabei muß lediglich berücksichtigt werden, daß nicht alle Tiere im ersten Jahr zur gleichen Zeit ihre Larvalphase beendeten und daher bis zum Winter unterschiedlich stark gewachsen waren. Da auch weiterhin kein völlig synchrones Wachstum erfolgt und auch der Zuwachs bei den Häutungen etwas variieren kann, verteilen sich die Tiere einer Generation im Laufe der Zeit über mehrere Größenklassen in Normalverteilung. Allerdings bewirkt die winterliche Häutungsruhe zunächst eine gewisse Synchronisation. Im Mai setzen bei steigenden Wassertemperaturen schlagartig Häutungen ein. Die Häutungsaktivität hält den Sommer hindurch an und wird erst bei den sinkenden Temperaturen im Herbst allmählich eingestellt.

Am Ende des Jahres 1975 waren alle Tiere der 74iger Generation geschlechtsreif. Die Weibchen hatten eine Größe von 25-35 mm und die Männchen von $35-45 \mathrm{~mm}$ erreicht. Dieses Wachstum entspricht einer Zahl von 4 bis 5 Häutungen.

Nach Erreichen der Geschlechtsreife häuten sich die Männchen und Weibchen zu verschiedenen Zeiten und unterschiedlich häufig. Sie unterscheiden sich deshalb etwas in ihrem Wachstum.

Im 3. Lebensjahr erreichten die Weibchen eine mittlere Carapaxbreite von 35 und die Männchen von 45-50 mm. Dies entspricht dem Zuwachs einer Häutung. Diese findet bei den Männchen vorwiegend in der Zeit von Mai bis Juni und bei den Weibchen nach Beendigung des Brutgeschäftes in der Zeit von Juli bis August statt.

Insgesamt haben die Krebse dann im Mittel 13 Wachstumshäutungen innerhalb von 3 Jahren durchgeführt. Hinzu kommen noch 5, in erster Linie morphogenetischen Veränderungen dienende Larvenhäutungen. Da die größten Weibchen $60 \mathrm{~mm}$ und die größten Männchen in der Schlei $75 \mathrm{~mm}$ breit waren, muß auch in den nachfolgenden Jahren noch geringes Wachstum erfolgen bzw. ein bis zwei weitere Häutungen stattfinden. Insgesamt kann daher für eine Strandkrabbengeneration in der Schlei unter günstigen Bedingungen mit einer maximalen Lebenszeit von 5 Jahren gerechnet werden.

Nach dem Erreichen der Geschlechtsreife werden alle Weibchen begattet. Dies geht aus dem großen Anteil trächtiger Weibchen in den Sommerfảngen von Juni bis August und aus hier nicht näher besprochenen Laboratoriumsuntersuchungen hervor. In diesen wurden alle nicht eiertragende Weibchen aus den Fängen mehrere Wochen isoliert von den Männchen in einem großen Aquarium gehalten. Alle diese Weibchen brachten innerhalb von $1 \frac{1}{2}$ Monaten Eier hervor.

In der Schlei gibt es nur eine Brutperiode im Sommer, in der einige Weibchen aber zweimal hintereinander ablaichen. Eine einmalige Laichsaison scheint allgemein in der Ostsee zu gelten, denn Rasmussen (1973) fand an der dänischen Ostseeküste übereinstimmend mit unseren Befunden nur eine Brutzeit von Mai bis Juli. Die meisten trächtigen Weibchen wurden im. Juni gefunden. Aus der Nordsee sind dagegen zwei Brutzeiten bekannt, eine im Winter, in der die Eier von November bis Dezember 
abgelaicht werden und eine zweite, weniger ausgeprägte im Sommer von Juni bis Juli (Broekhuysen, 1937).

In der Literatur wird mehrfach darauf hingewiesen, daß die trächtigen Weibchen in tieferes Wasser abwandern. Dies gilt nicht nur für die Nordsee (Broekhuysen, 1937), sondern auch für die Ostsee (Rasmussen, 1973; Thorson, 1946). Für die Schlei konnten wir kein ausgeprägtes Wanderverhalten der trächtigen Weibchen feststellen. Allerdings waren diese Tiere vermehrt im äußeren, tiefen Teil der Schlei zu finden.

Neben der durch die Trächtigkeit bedingten Wanderung der Weibchen kommt es im Winter zu einer allgemeinen Abwanderung sämtlicher Tiere aus den flacheren Randgebieten der Schlei in das tiefe Rinnensystem, wo sie nach unseren Beobachtungen in dem beschriebenen Spaltensystem während des Winters nahezu bewegungslos verbleiben. Abwanderungen von C. maenas im Winter aus dem Flachwasser in tiefere und wärmere sowie salzreichere Gebiete sind aus der Ostsee und der Nordsee bekannt (Broekhuysen, 1937; Poulsen, 1949; Muus, 1967; Rasmussen, 1973). Anscheinend sind die Strandkrabben durch tiefe Temperaturen gefährdet. Untersuchungen von Poulsen (1949) ergaben in den extremen Eiswintern (1940-1942) ein Massensterben von $C$. maenas in flachen Küstengewässern.

Eine Abwanderung aus der Schlei in die Ostsee wegen ungüstiger Umweltbedingungen kann nach unseren Untersuchungen ausgeschlossen werden.

Die starken Populationsschwankungen von C. maenas in der Schlei lassen sich auf wechselnde hydrographische Bedingungen in den verschiedenen Jahren zurückführen, die vor allem die empfindliche Larvalentwicklung entscheidend beeinflussen. Wie entsprechende Laboruntersuchungen gezeigt haben (Dries \& Adelung, 1976) besitzen die Larven nur ein enges Toleranzspektrum für Temperatur und Salzgehalt. In Laboratoriumsexperimenten entwickelten sich die Larven am besten bei $18 \%$ und $15{ }^{\circ} \mathrm{C}$ bzw. $28 \%$ und $12,5^{\circ} \mathrm{C}$. Bei einem Salzgehalt von $13 \%$ starben die Larven bei allen erprobten Temperaturen.

Während der Fortpflanzungsperiode im Jahre 1974 entsprachen die Salinität in der Schlei mit $15-17 \%$ und die Temperatur mit $14-17^{\circ} \mathrm{C}$ diesen günstigen Bedingungen. 1975 bestanden dagegen mit Salzgehalten von 12,4 bis $13,3 \%$ und Temperaturen von $20-21^{\circ} \mathrm{C}$ ungünstige Bedingungen für die Entwicklung von Larven, so daß sich daraus die auf die 1974iger Generation folgende zahlenschwache Generation 1975 erklärt.

Ähnliche Bedingungen haben wahrscheinlich auch zu den vorangegangenen nachwuchsschwachen Jahrgängen 1971-1973 geführt. Zwar liegen hier keine direkten Daten über Salzgehalte und Temperatur in der Schlei vor, aber sie lassen sich aus den allgemeinen hydrographischen Bedingungen der Ostsee ableiten. So waren im Fehmarn-Belt die Oberflächensalzgehalte in dem nachwuchsreichen Jahr 1974 höher als 1975. In den Jahren 1971-1973 waren die Salzgehalte dort z.Zt. der Larvenphase von C. maenas ausgesprochen niedrig. Die zu dieser Zeit herrschenden meteorologischen Verhältnisse ließen wahrscheinlich nur salzarmes Oberflächenwasser aus der Ostsee in die Schlei eindringen, wodurch die Fortpflanzung von C. maenas mehrere Jahre hintereinander so gehemmt war, daß zu Beginn des Jahres 1974 nur noch wenige große Krebse vorhanden waren. Im Winter 1974/75 wurden dagegen nach dem für die Larven günstigen Sommer 1974 zahlreiche juvenile Tiere gefunden.

1976 lagen in dem Mündungsgebiet der Schlei bei gleichem Salzgehalt wie in dem davorliegenden Gebiet der Ostsee die Temperaturen in der Schlei während der Brut- 
und Larvalzeit von C. maenas um $1,6^{\circ} \mathrm{C}$ höher. Wahrscheinlich bedingt diese höhere Temperatur, da $\beta$ in der Schlei die Larven früher als in der Ostsee auftreten. Dies beweist gleichzeitig, daß sich $C$. maenas in der Schlei entwickeln kann und dort eine eigene Population vorhanden ist.

Das Mündungsgebiet der Schlei bietet offensichtlich trotz der vor allem in Richtung Kappeln zunehmenden Verschmutzung (Rheinheimer, 1970; Rieper, 1976) günstige Lebensmöglichkeiten für die Larven. Dies geht u.a. daraus hervor, daß bei den meisten in der Schlei gefangenen Larven der Darm gefüllt war, während in dem davorliegenden Gebiet der Ostsee wesentlich weniger Larven eine Magenfüllung aufwiesen.

Daß die Larvalentwicklung von $C$. maenas in hohem Maße von den gerade herrschenden hydrographischen Bedingungen abhängt, geht auch aus der Untersuchung anderer Autoren hervor. So fing Kändler (1961) in der Kieler Förde in windschwachen Sommern mit anhaltender Hochdruckwetterlage und dadurch verursachtem geringem Salzgehalt des Wassers sehr wenige Larven, in Jahren mit hohem Salzgehalt, verursacht durch vorherrschende Westwinde dagegen viele. Ahnliche Schwankungen in der Bestandsdichte ließen sich auch im Isefjord feststellen (Rasmussen, 1973). Von 1941-1967 wurden 7 Jahre mit Nachwuchs und 4 ohne Nachwuchs registriert. Während der pelagischen Phase sank in den 7 Jahren mit Nachwuchs die Temperatur nicht unter $15,6^{\circ} \mathrm{C}$ und der Salzgehalt nicht unter $18,2 \%$. Für die Schlei kann man annehmen, daß bei Salzgehalten von 15-17\% und Temperaturen von $14-17{ }^{\circ} \mathrm{C}$ ausreichende Bedingungen für eine gesicherte Fortpflanzung bestehen. Darunter liegende Salzgehalte, möglicherweise durch höhere Temperaturen in ihrem ungünstigen Einfluß verstärkt, verhindern oder beeinträchtigen den Nachwuchs.

Während für die Existenz der Larven sowohl die Faktoren Salzgehalt als auch Temperatur entscheidend sind, wirkt sich für die Jungtiere und die adulten Krebse lediglich die Temperatur in extremen Wintern negativ auf den Bestand aus. So stellte Poulsen (1949) fest, daß in Flachwassergebieten die Fänge nach sehr kalten Wintern mit Eisbildung zurückgingen, daß aber in Tiefen zwischen 7 und $12 \mathrm{~m}$ die gleiche Zahl von Tieren wie in wärmeren Wintern gefangen werden kann. Auch die Fisscher in der Schlei fingen 1972 nicht weniger Krebse als in wärmeren Wintern, obwohl in dem Winter 1971/ 72 die Schlei 6 Wochen lang eine geschlossene Eisdecke trug. Man kann daher annehmen, daß C. maenas in der Schlei kaum durch besonders kalte Winter gefährdet ist. Hierzu tragen sicher auch die günstigen Bodenverhältnisse mit den idealen Unterschlupfmöglichkeiten bei, die die Krebse im Winter beziehen.

Zusammenfassend kann man feststellen, daß die Schlei, insbesondere das Mündungsgebiet, für die Strandkrabben bei günstigen hydrographischen Verhältnissen ein sehr geeignetes Biotop darstellt. Die Tiere finden im Winter gute Bedingungen; sich vor extremen Temperaturen, der Verfolgung durch Feinde und zu starker Strömung zu schützen. In den warmen Jahreszeiten finden sowohl die Larven als auch die juvenilen und adulten Tiere gute Nahrungsbedingungen. Weiterhin bleiben die Strandkrabben in der Schlei von dem sonst oft sehr häufig in anderen Biotopen zu findenden Parasiten Sacculina carcini verschont, da sich dessen Larven wegen des niedrigen Salzgehaltes in der Schlei nicht entwickeln können. Daher kommt es unter günstigen Umweltbedingungen schnell zu einem sprunghaften Ansteigen der Population. Dies war im Jahr 1974 der Fall: Offensichtlich ist die Strandkrabbe in der Lage, nach mehreren Jahren der Abwesenheit im Ókosystem ihren Platz sehr rasch zurückzuerobern. 
Nach den Untersuchungen in der Schlei dürften hier die Temperatur und der Salzgehalt die entscheidenden begrenzenden abiotischen Faktoren darstellen. In gewissen geographischen Regionen mag die Temperatur der dominierende Faktor sein, aber in der Schlei sowie vermutlich in der gesamten Ostsee dürfte vor allem der Salzgehalt der Hauptfaktor für die Lebens- und Fortpflanzungsmöglichkeiten von C. maenas sein. Die Temperatur spielt hier insofern eine Rolle, als sie den negativen Einfluß von geringem Salzgehalt in gewissen Grenzen verstärken kann.

\section{ZITIERTE LITERATUR}

Braun, M., 1888. Faunistische Untersuchungen in der Bucht von Wismar. - Arch. Ver. Freunde Naturg. Mecklenb. 42, 57-84.

Broekhuysen, G. J., Jr., 1937. On the development and distribution of Carcinus maenas (L.). - Archs néerl. Zool. 2, 257-382.

Dries, M. \& Adelung, D., 1976. Neue Ergebnisse über die Aufzucht von Carcinus maenas im Laboratorium. - Mar. Biol. 38, 17-24.

Kändler, R., 1952. Jahreszeitliche Vorkommen und unperiodisches Auftreten von Fischbrut, Medusen und Dekapodenlarven im Fehmarnbelt in den Jahren 1934-1943. - Ber. dt. wiss. Kommn Meeresforsch. 12, 49-85.

Kändler, R., 1961. Über das Vorkommen von Fischbrut, Decapodenlarven und Medusen in der Kieler Förde. - Kieler Meeresforsch. 17, 48-64.

Muus, B. J., 1967. The fauna of Danish estuaries and lagoons. - Meddr Danm. Fisk.-og Havunders. 5 (1), 1-316.

Poulsen, E. M., 1949. On the distribution of the Brachyura (Crustacea Decapoda) in Danish waters. Vidensk. Meddr dansk naturh. Foren. 111, 111-130.

Rasmussen, E., 1973. Systematics and ecology of the Isefjord marine fauna (Denmark). - Ophelia 11, $1-507$.

Rheinheimer, G., [Hrsg.], 1970. Chemische, mikrobiologische und planktologische Untersuchungen in der Schlei im Hinblick auf deren Abwasserbelastungen. - Kieler Meeresforsch. 26, 105-215.

Rieper, M., 1976. Investigations in the relationship between algal blooms and bacterial populations in the Schlei Fjord (western Baltic Sea). - Helgoländer wiss. Meeresunters. 28, 1-18.

Ropes, J. W., 1968. The feeding habits of the green crab. Carcinus maenas (L.). - Fish. Bull. U. S. 67, 183-203.

Schellenberg, A., 1928. Krebstiere oder Crustacea. II. Decapoda, Zehnfüßer. - Tierwelt Dtl. 10, $127-130$.

Schulz, S., 1939. Das Makrobenthos der südlichen Beltsee. - Beitr. Meeresk. 26, 21-46.

Thorson, G., 1946. Reproduction and larval development of Danish marine bottom invertebrates, with special reference to the planktonic larvae in the sound (Øresund). (11. Crustacea). - Meddr Kommn Danm Fisk.-og Havunders. (Plankton) 4, (1) 318-343. 